\title{
Development of an Educational Robotic Training Kit.
}

\begin{abstract}
Robotics is an applied science subject in engineering course that concerning control and automation in manufacturing industry now. Engineering students are forced to learn all theories and formulas that encompass the areas of mathematics, physics, micro-electronics, dynamics and kinematics, control systems, human factors, and manufacturing operation in a single subject without any hands-on practical skills. This paper provides an idea to break the barriers of conventional lecturing and laboratory exercising for engineering students with new development of educational robotic kit. This practical approach is able to support low cost robotics course and offers simple, time saving instruction outcomes to engineering students in the kinematics study of robot manipulators with consideration of structure design, serial servo circuit board, microcontroller programming, and graphical user interface (GUI) of PC controller. General architecture of 3-degree-of-freedom (DOF) robot manipulator with two links can be solved easily with the help of this robotic kit.
\end{abstract}

Keyword: Robotic; Robot; Manipulator; Engineering; Education; Training 